\title{
Inverse Prediction and Application of Homotopy Perturbation Method for Efficient Design of an Annular Fin with Variable Thermal Conductivity and Heat Generation
}

\author{
Ashis Mallick ${ }^{a}$, Rajiv Ranjan ${ }^{a}$, Dilip K. Prasad ${ }^{b}$ and \\ Ranjan Das ${ }^{c}$
}

${ }^{a}$ Indian Institute of Technology (ISM) Dhanbad

Department of Mechanical Engineering, Dhanbad 826004, India

${ }^{b}$ Nanyang Technological University

School of Computer Science and Engineering, Singapore 639798

${ }^{c}$ Indian Institute of Technology Ropar

School of Mechanical, Materials and Energy Engineering, Punjab 140001, India

E-mail(corresp.): mal1230yahoo.com

Received September 10, 2015; revised July 25, 2016; published online September 15, 2016

\begin{abstract}
In the present work, various thermal parameters of an annular fin subjected to thermal loading are inversely estimated using differential evolution (DE) method. In order to obtain the temperature field, the second order nonlinear differential equation for heat transfer with variable thermal conductivity and internal heat generation is solved using Homotopy Perturbation Method (HPM). Classical thermoelasticity approach coupled with an HPM solution for temperature field is employed for the forward solution of thermal stresses. It is interesting that the internal heat generation does not affect the radial stresses, while the temperature field and the tangential stresses are influenced by the heat generation parameters. As the tangential stresses are mainly responsible for mechanical failure due to thermal loading in an annular fin, the unknown thermal parameters are inversely estimated from a prescribed tangential stress field. The reconstructed stress fields obtained from the inverse parameters are found to be in good agreement with the actual solution.
\end{abstract}

Keywords: heat conduction, inverse problem, nonlinear problem, perturbation method, mathematical model.

AMS Subject Classification: 65H20; 31A25; 80A20.

\section{Introduction}

In todays engineering development, heat dissipation has become an important concern. There are different modes to enhance heat transfer. Among them, 
extended surface is one mode of heat transfer which has wide application for industrial purposes. Kraus et al. [19] presented an extensive review on heat transfer in extended surfaces. Fin has effective application in the case of natural convection and it is always applied towards less heat transfer coefficient. In this regard, the increase of surface area helps to decrease the convective thermal resistance. A comprehensive literature review reveals that most of the heat transfer studies neglect the influence of internal heat generation. This consideration certainly simplifies the mathematical formulation of heat transfer. However, because of heat conduction, the contribution of internal heat generation cannot be ignored and it varies with temperature in a real situation. Furthermore, due to large temperature variation from the base to the tip of the fin, the thermal conductivity varies with the temperature.

Cumo et al. [13] first considered the internal heat generation for two dimensional heat transfer analysis of a straight fin of general profile. In their analysis, computationally efficient numerical approach, Gauss-Seidel iteration method was employed. Later, the internal heat generation along with the variable heat transfer coefficient and variable thermal conductivity was taken into account for longitudinal fins of various profiles by Melese and Wilkins [23].

The properties of thermal parameters play an important role in transfer of heat from the hot surface to the cold surface. In many equipment and industrial application such as motor vehicle, air conditioning system, electronics equipment and refineries, there is a need of efficient heat transfer. Thus, many researchers had concentrated on the improvement of thermal efficiency through the enhancement of heat transfer [7,26].

Most of the closed form solutions for heat transfer in a conducting convecting fin are solved with an assumption of constant thermal parameters. However, physically the heat transfer phenomena are nonlinear. Thus, obtaining a closed form solution emulating a real situation of heat transfer through fin is extremely difficult. Due to this reason, the researchers prefer to put their effort in numerical solution or semi analytical solution of heat transfer problems. An extensive literature survey reveals that various numerical methods and semi analytical approaches such as the least squares method (LSM), variational iteration method (VIM), Adomian decomposition method (ADM), differential transform method (DTM), homotopy analysis method (HAM), homotopy perturbation method (HPM) have been employed to solve the nonlinear heat transfer equation of fin $[10,11,12,24]$. Some of the recent works related to the solution of nonlinear heat transfer equation of the fin are presented in the following section.

Recently, Aziz and Bouaziz [4] applied LSM to study the temperature distribution in a longitudinal fin with temperature dependent internal heat generation and thermal conductivity. Singla and Das [25] employed ADM to solve the heat transfer equation for a straight rectangular conductive-convective fin with linearly variable heat generation and thermal conductivity. In their study, an inverse method was applied for predicting the unknown thermal parameters and the heat generation number from the forward solution of the temperature field. Optimum profile of thin fins with volumetric heat generation was obtained by considering different profiles by Kundu and Das [20]. It may be noted that a 
constant heat generation rate throughout the fin volume was assumed in their analysis. The temperature distribution due to heat transfer in a porous fin $\left(\mathrm{Si}_{3} \mathrm{~N}_{4}\right.$ and $\mathrm{Al}$ ) with temperature dependent heat generation was studied by Hatami et al. [16]. In their analysis, three different mathematical approaches, DTM, Collocation method (CM) and LSM were employed and compared.

It is well known that the non-uniform temperature distribution is mainly responsible to develop thermal stresses in the fin, and consequently material failures such as crack propagation, delamination, fatigue, creep occur in the fin material. In spite of that critical issue, very few researchers addressed the effect of thermal stresses in fin analysis. Some of them are Wu [29], Yang and Chu [30], Chiu and Chen [9], and Mallick et al. [22]. The objective of their study was to evaluate the thermal stresses in an annular fin by solving the governing equation of heat transfer coupling with the thermo-elasticity equation and proper boundary conditions. Such type of solution is well-posed and referred to as the forward or direct problem. However, the challenge is to estimate the optimal parameters and material properties, and therefore solving the inverse problem is of greater importance for efficient designing of the fin. A group of authors presented inverse solution approaches to estimate the unknown parameters of fin $[8,14]$. However, their analyses were limited to heat transfer, and thereafter temperature distribution only. They estimated the unknown parameters inversely from a predefined temperature distribution. Recently, Mallick and Das [21] have presented an inverse analysis to predict the unknown parameters of an annular fin subjected to thermal stress. In their analysis, the internal heat generation was neglected and constant thermal conductivity was assumed. A simple perturbation method coupled with thermo-elasticity equation was employed for the forward solution of thermal stresses, and then simplex search method was adopted for the inverse solution.

The above literature review shows that no effort yet has been made towards the inverse as well as forward analysis of an annular fin having variable thermal conductivity and internal heat generation. The aim of this study is an inverse estimation of unknown thermal parameters for an annular fin subjected to thermal stresses. In this regard, first the forward solution for temperature field of an annular fin with variable thermal conductivity and internal heat generation is obtained using HPM, which is a semi-analytical approach. The solution of temperature field is then coupled with the thermo-elasticity solution for stress field. Differential evolution (DE) method is employed for the inverse prediction of unknown thermo-mechanical parameters. The stress field is reconstructed using various combinations of thermal parameters obtained from the inverse solution. It has been observed that the reconstructed stress fields is in a very good agreement with the actual solution.

Next we give the nomenclature, used in the text: $r_{i}, r_{0}, t$ defines inner radius, outer radius and thickness of the fin, $h$ is coefficient of convective heat transfer, $k(T)$ denotes thermal conductivity, $k_{0}, q_{0}$ are thermal conductivity and internal heat generation at ambient temperature, $\kappa$ describes the variation of thermal conductivity, $\beta$ is non-dimensional parameter describing the variation of thermal conductivity, $N$ is non-dimensional thermo-geometric parameter, $\left(\frac{2 h r_{i}{ }^{2}}{k_{0} t}\right)^{0.5}, G=q_{0} r_{i}^{2} / k_{0}\left(T_{b}-T_{\infty}\right)$ is non-dimensional heat generation 
parameter, $\varepsilon$ is parameter describing the variation of heat generation, $E_{G}$ is non-dimensional parameter describing the variation of heat generation, $T_{b}$ defines the base temperature of fin, $T_{\infty}$ is ambient temperature, $r, \phi$ are polar coordinates, $\alpha$ is linear coefficient of thermal expansion, $E$ is Young's modulus, $\chi$ is dimensionless coefficient of thermal expansion, $\alpha\left(T_{b}-T_{\infty}\right), \nu$ is Poisson's ratio, $\varepsilon_{r}, \varepsilon_{\phi}$ are radial and tangential strain, $\bar{\sigma}_{r}, \bar{\sigma}_{\phi}$ are dimensionless radial and tangential stress, $\sigma_{r} / E, \sigma_{\phi} / E, \xi=\frac{r-r_{i}}{r_{i}}$ is dimensionless radius of fin, $\xi_{1}=\xi+1$ is dimensionless radius of fin, $R=r_{0} / r_{i}$ is dimensionless outer radius, $\theta=\frac{\left(T-T_{\infty}\right)}{\left(T b-T_{\infty}\right)}$ is dimensionless temperature, $p$ is imbedding parameter, $\eta$ is fin efficiency, $Q_{f}$ is actual heat transfer, $Q_{\max }$ is the maximum possible heat transfer, $F(\xi)$ is an objective function, $\sigma_{\phi_{j}}(n)$ is a guessed tangential stress field.

\section{Problem Formulation}

\subsection{Description of the problem}

Consider an axisymmetric annular fin of uniform thickness, $t$, inner radius, $r_{i}$, and outer radius, $r_{0}$. The fin is made up of homogeneous isotropic material as shown in Figure 1. The fin's surfaces are exposed in a convective environment at temperature, $T_{\infty}$ and its base is attached to a surface with fixed temperature $T_{b}$. The radius of the fin is very large as compared to its thickness. Hence, the fin's tip is assumed to be insulated due to the negligible heat transfer through its tip. Due to axisymmetric nature and small thickness, the heat flow is considered to be in radial direction $r$ only. For one dimensional heat transfer, the law of conservation of energy gives the following energy balance equation [19]

$$
t \frac{d}{d r}\left[k(T) r \frac{d T}{d r}\right]-2 h r\left(T-T_{\infty}\right)+q(T) t r=0
$$

where $T$ represents the temperature, $h$ is the convective heat transfer coefficient, $k(T)$ is variable thermal conductivity, and $q(T)$ represents the internal heat generation. The variation of thermal conductivity and internal heat generation are assumed to be linear with temperature as:

$$
k(T)=k_{0}\left\{1+\kappa\left(T-T_{\infty}\right)\right\}, \quad q(T)=q_{0}\left\{1+\varepsilon\left(T-T_{\infty}\right)\right\},
$$

where $k_{0}$ and $q_{0}$ are thermal conductivity and internal heat generation at the ambient temperature respectively. The parameters, $\kappa$ and $\varepsilon$ represent the variation of thermal conductivity and internal heat generation. Assume that the thickness of the fin wall is very small. Thus, employing zero conductive thermal resistance in the fin wall, the following boundary conditions can be obtained for the energy balance equation,

$$
r=r_{i}: \quad T=T_{b}, \quad r=r_{0}: \frac{d T}{d r}=0 .
$$




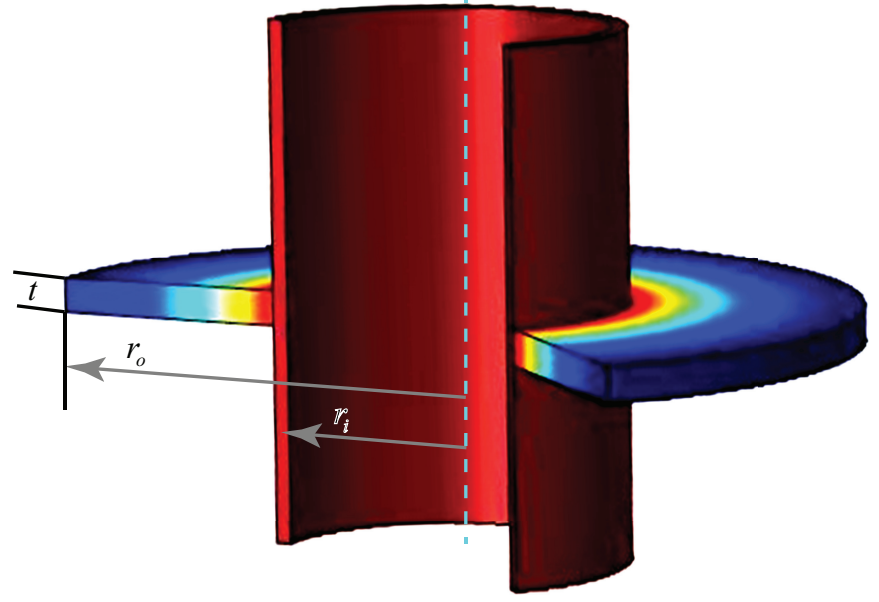

Figure 1. Geometry of an annular fin.

For simplicity and convenience in the analysis, various non-dimensional parameters are introduced as,

$$
\begin{aligned}
& \theta=\frac{T-T_{\infty}}{T_{b}-T_{\infty}}, \quad B_{i}=\frac{h r_{i}}{k_{0}}, \quad \beta=\kappa\left(T_{b}-T_{\infty}\right), \quad E_{G}=\varepsilon\left(T_{b}-T_{\infty}\right), \\
& \xi=\frac{r-r_{i}}{r_{i}}, \quad R=\frac{r_{0}}{r_{i}}, \quad N^{2}=\frac{2 h r_{i}^{2}}{k_{0} t}, \quad G=\frac{q_{0} r_{i}^{2}}{k_{0}\left(T_{b}-T_{\infty}\right)} .
\end{aligned}
$$

The use of aforementioned nondimensional parameters (i.e (2.1)) reduces the governing equation and boundary conditions into the following nondimensional form:

$$
\begin{aligned}
& \frac{d^{2} \theta}{d \xi^{2}}+\beta \theta \frac{d^{2} \theta}{d \xi^{2}}+\beta \theta \frac{1}{1+\xi} \frac{d \theta}{d \xi}+\frac{1}{1+\xi} \frac{d \theta}{d \xi}+\beta\left(\frac{d \theta}{d \xi}\right)^{2}-N^{2} \theta+G\left(1+E_{G} \theta\right)=0 . \\
& \xi=0: \quad \theta=1, \quad \xi=R-1: \quad \frac{d \theta}{d \xi}=0 .
\end{aligned}
$$

\subsection{Homotopy Perturbation Method (HPM)}

HPM is a relatively new mathematical technique for solving linear and nonlinear differential equation efficiently. This method was first introduced by J.H. $\mathrm{He}[17,18]$ and is extensively used now-a-days for nonlinear initial and boundary value problems. In this method, the standard homotopy and perturbation are merged to take full advantage of homotopy and perturbation by introducing an embedding parameter instead of small variational parameters in an equation. Using this method, one can obtain an approximate closed form solution of the nonlinear equation without linearizing the problem. The method converges very fast with high accuracy $[3,5]$. In order to understand HPM, consider a general nonlinear differential equation as follows:

$$
L(u)+N(u)-f(r)=0, \quad r \in \Omega
$$


with boundary conditions,

$$
B\left(u, \frac{\partial u}{\partial n}\right)=0 \quad \text { at } \quad r \in \Gamma
$$

where $L$ and $N$ are linear and nonlinear operators respectively, $f(r)$ is known as analytical function, $B$ is a boundary operator, $\Gamma$ is the boundary of the domain $\Omega$, and $\frac{\partial}{\partial n}$ represents the directional derivatives in outward normal direction to $\Omega$.

The homotopy may be constructed as,

$$
H(v, p)=(1-p)\left[L(v)-L\left(u_{0}\right)\right]+p[L(v)+N(v)-f(r)]=0
$$

where $p \quad(p \in[0,1])$ is the artificial embedded parameter which always changes from zero to one and $u_{0}$ is an initial approximation which satisfies the boundary conditions. The $(2.3)$ becomes $H(v, 0)=\left[L(v)-L\left(u_{0}\right)\right]=0$ and $H(v, 1)=$ $L(v)+N(v)-f(r)=0$ when parameter, $p$ is 0 and 1 respectively. The solutions of $(2.3)$ can be approximated as a power series of $p$,

$$
v=v_{0}+p v_{1}+p^{2} v_{2}+p^{3} v_{3}+\ldots
$$

The values of $v_{0}, v_{1}, v_{2}, v_{3}, \ldots$ can be determined analytically. The best approximation is obtained by putting $p=1$ :

$$
u=\lim _{p \rightarrow 1} v=v_{0}+v_{1}+v_{2}+v_{3}+\ldots
$$

\subsection{Fin temperature calculation}

In order to solve the governing equation of heat transfer, homotopy is constructed for (2.2) in a manner similar to (2.3) as follows:

$$
(1-p)\left[L(\theta)-L\left(\theta_{0}\right)\right]+p[L(\theta)+N(\theta)-f(r)]=0, \quad p \in[0,1] .
$$

Here $L=\frac{d^{2}}{d \xi^{2}}, \theta_{0}$ is the initial approximation and $\theta$ is a function of $\xi$. Now, (2.2) can be expressed in the following HPM form:

$$
\begin{gathered}
L(\theta)+p L\left(\theta_{0}\right)-L\left(\theta_{0}\right)=-p\left[\beta \theta \frac{d^{2} \theta}{d \xi^{2}}+\beta \theta \frac{1}{1+\xi} \frac{d \theta}{d \xi}+\frac{1}{1+\xi} \frac{d \theta}{d \xi}\right. \\
\left.+\beta\left(\frac{d \theta}{d \xi}\right)^{2}-N^{2} \theta+G\left(1+E_{G} \theta\right)\right] .
\end{gathered}
$$

Similar to (2.4), the solution of (2.5) has the form of power series of $p$ as,

$$
\theta=\theta_{0}+p \theta_{1}+p^{2} \theta_{2}+\ldots
$$


Substituting the value of $\theta$ from (2.6) into (2.5) and equating the terms of identical powers of $p$ gives the following:

$$
\begin{aligned}
& p^{0}: \theta_{0}=\theta_{0} \text { with BCs } \xi=0: \theta_{0}=1 \text { and } \xi=R-1: \frac{d \theta_{0}}{d \xi}=0, \\
& p^{1}: \frac{d^{2} \theta_{1}}{d \xi^{2}}=-G\left(1+E_{G}\right)+N^{2} \text { with BCs } \\
& \xi=0: \theta_{1}=0 \text { and } \xi=R-1: \frac{d \theta_{1}}{d \xi}=0, \\
& p^{2}: \frac{d^{2} \theta_{2}}{d \xi^{2}}=-\beta\left\{\theta_{0} \frac{d^{2} \theta_{1}}{d \xi^{2}}+\frac{1}{1+\xi} \theta \frac{d \theta_{1}}{d \xi}\right\}-\frac{1}{1+\xi} \frac{d \theta_{1}}{d \xi}-G E_{G} \theta_{1}+N^{2} \theta_{1} \\
& \text { with BCs } \xi=0: \theta_{2}=0 \text { and } \xi=R-1: \frac{d \theta_{2}}{d \xi}=0 .
\end{aligned}
$$

Solving (2.7), (2.8), (2.9), the following approximate closed form solution for temperature field is obtained from (2.6) considering $p=1$ :

$$
\begin{aligned}
\theta= & 1-\left\{G\left(1+E_{G}\right)-N^{2}\right\}\left\{\xi^{2} / 2-(R-1) \xi\right\}-\beta \theta_{1} \\
& -(\beta+1)\left\{G\left(1+E_{G}\right)-N^{2}\right\}\left[R\{(1+\xi) \ln (1+\xi)-(1+\xi)\}-\xi^{2} / 2\right] \\
& +\left(N^{2}-E_{G} G\right)\left\{G\left(1+E_{G}\right)-N^{2}\right\}\left\{(R-1) \frac{\xi^{3}}{6}-\frac{\xi^{4}}{24}\right\}+c_{1} \xi+c_{2},
\end{aligned}
$$

where $c_{1}$ and $c_{2}$ are the integral constants. The constants $c_{1}$ and $c_{2}$ are evaluated from the boundary conditions of (2.9) as follows:

$$
\begin{aligned}
c_{1}= & (\beta+1)\left\{G\left(1+E_{G}\right)-N^{2}\right\}\{R \ln R-(R-1)\} \\
& -\left(N^{2}-E_{G} G\right)\left\{G\left(1+E_{G}\right)-N^{2}\right\}(R-1)^{3} / 3, \\
c_{2}= & -(\beta+1)\left\{G\left(1+E_{G}\right)-N^{2}\right\} R .
\end{aligned}
$$

\subsection{Thermal stresses}

It is evident that the stress distribution in an axisymmetric annular thin fin varies along the radial direction only. Neglecting the body force and inertia, the equation of equilibrium for axisymmetric plane stress condition in polar coordinate system is given as [28],

$$
r \frac{d \sigma_{r}}{d r}+\sigma_{r}-\sigma_{\phi}=0
$$

where $\sigma_{r}$ and $\sigma_{\phi}$ are radial and tangential stresses respectively. Let us introduce a stress function, $\psi$, such that

$$
\sigma_{r}=\psi / r \text { and } \sigma_{\phi}=d \psi / d r
$$

(2.11) identically satisfies the equation of equilibrium. The strain-displacement relationship can be expressed as [6],

$$
\varepsilon_{r}=\frac{d u}{d r} \text { and } \varepsilon_{\phi}=\frac{u}{r},
$$


where $u$ is the radial displacement. (2.12) gives the following strain compatibility equation,

$$
r \frac{d \varepsilon_{\phi}}{d r}+\varepsilon_{\phi}-\varepsilon_{r}=0
$$

Following Hooke's law for plane stress condition, the stress-strain relationship is given as,

$$
\varepsilon_{r}=\frac{1}{E}\left(\sigma_{r}-\nu \sigma_{\phi}\right)+\alpha T \text { and } \varepsilon_{\phi}=\frac{1}{E}\left(\sigma_{\phi}-\nu \sigma_{r}\right)+\alpha T
$$

where $E, \nu$ and $\alpha$ are the modulus of elasticity, Poisson's ratio and coefficient of thermal expansion respectively. Substituting (2.14) and (2.11) into (2.13) yields the following compatibility equation in terms of the stress function,

$$
\frac{1}{d r}\left[\frac{1}{r} \frac{d}{d r}(r \psi)\right]=-\alpha E \frac{d T}{d r} .
$$

The solution of the above equation for $\psi$ yields,

$$
\psi=-\frac{\alpha E}{r} \int_{r_{i}}^{r}\left(T-T_{\infty}\right) r d r+C_{1} r+C_{2} / r
$$

where $C_{1}$ and $C_{2}$ are the constants of integration which can be estimated from the boundary conditions. The inner and outer surfaces of the fin are considered to be traction free, and hence the radial stress, $\sigma_{r}=0$ at $r=r_{i}$ and $r_{0}$. Employing these boundary conditions, the constant of integrations are obtained using (2.11) and (2.15) as follows,

$$
C_{1}=\frac{\alpha E}{r_{0}^{2}-r_{i}^{2}} \int_{r_{i}}^{r_{0}}\left(T-T_{\infty}\right) r d r, \quad C_{2}=-\frac{r_{i}^{2} \alpha E}{r_{0}^{2}-r_{i}^{2}} \int_{r_{i}}^{r_{0}}\left(T-T_{\infty}\right) r d r .
$$

Now, the stress components can be evaluated directly from (2.11),

$$
\begin{aligned}
\sigma_{r} & =-\frac{\alpha E}{r^{2}} \int_{a}^{r}\left(T-T_{\infty}\right) r d r+\frac{\alpha E}{r_{0}^{2}-r_{i}^{2}}\left(1-\frac{r_{i}^{2}}{r^{2}}\right) \int_{r_{i}}^{r_{0}}\left(T-T_{\infty}\right) r d r, \\
\sigma_{\phi} & =-\alpha E\left(T-T_{\infty}\right)+\frac{\alpha E}{r^{2}} \int_{r_{i}}^{r}\left(T-T_{\infty}\right) r d r+\frac{\alpha E}{r_{0}^{2}-r_{i}^{2}}\left(1+\frac{r_{i}^{2}}{r^{2}}\right) \int_{r_{i}}^{r_{0}}\left(T-T_{\infty}\right) r d r .
\end{aligned}
$$

The above stress equations can be expressed in the following dimensionless form

$$
\begin{aligned}
& \bar{\sigma}_{r}=-\frac{\chi}{\xi_{1}^{2}} \int_{1}^{\xi_{1}} \theta \xi_{1} d \xi_{1}+\frac{\chi\left(\xi_{1}^{2}-1\right)}{\left(R^{2}-1\right) \xi_{1}^{2}} \int_{1}^{R} \theta \xi_{1} d \xi_{1} \\
& \bar{\sigma}_{r}=-\chi \theta+\frac{\chi}{\xi_{1}^{2}} \int_{1}^{\xi_{1}} \theta \xi_{1} d \xi_{1}+\frac{\chi\left(\xi_{1}^{2}+1\right)}{\left(R^{2}-1\right) \xi_{1}^{2}} \int_{1}^{R} \theta \xi_{1} d \xi_{1}
\end{aligned}
$$

where the various dimensionless entities are

$$
\bar{\sigma}_{r}=\frac{\sigma_{r}}{E}, \quad \bar{\sigma}_{\phi}=\frac{\sigma_{\phi}}{E}, \quad \xi_{1}=\frac{r}{r_{i}}, \quad \chi=\alpha\left(T_{b}-T_{\infty}\right) .
$$


The relationship between two dimensionless radii $\xi$ and $\xi_{1}$ is $\xi_{1}=\xi+1$. For consistency in the solution, the stress field can be expressed in the same nondimensional radius $\xi$, as used in temperature field. Thus, the stress field equations now can be expressed in the following form

$$
\begin{aligned}
& \bar{\sigma}_{r}=-\frac{\chi}{(\xi+1)^{2}} \int_{0}^{\xi} \theta(\xi+1) d \xi+\frac{\chi\left(\xi^{2}+2 \xi\right)}{\left(R^{2}-1\right)(\xi+1)^{2}} \int_{0}^{R-1} \theta(\xi+1) d \xi \\
& \bar{\sigma}_{\phi}=-\chi \theta+\frac{\chi}{(\xi+1)^{2}} \int_{0}^{\xi} \theta(\xi+1) d \xi+\frac{\chi\left(\xi^{2}+2 \xi+2\right)}{\left(R^{2}-1\right)(\xi+1)^{2}} \int_{0}^{R-1} \theta(\xi+1) d \xi
\end{aligned}
$$

\subsection{Fin efficiency}

The fin efficiency is defined as a parameter to measure the thermal performance. It is the ratio of the actual heat dissipation of a fin to the maximum possible heat dissipation if the entire fin surface is at the same temperature as its base. The efficiency for an annular fin is formulated in the non-dimensional form as $[2]$

$$
\eta=\frac{Q_{f}}{Q_{\max }}=\frac{4 \pi h \int_{r_{a}}^{r_{b}}\left(T-T_{\infty}\right) r d r}{2 \pi h\left(r_{2}^{2}-r_{1}^{2}\right)\left(T_{b}-T_{\infty}\right)}=\frac{2 \int_{0}^{R-1}(1+\xi) \theta d \xi}{\left(R^{2}-1\right)}
$$

where $Q_{f}$ and $Q_{\max }$ are the actual heat transfer rate and maximum possible heat transfer respectively.

\subsection{Inverse technique using differential evolution}

The differential evolution (DE) is a fast and reasonably robust populationbased search method proposed by Storn and Price [27]. This method is used to optimize the unknown parameters iteratively in various field of engineering. As compared to most other evolutionary methods which do not use any form of deterministic update, DE's update scheme includes a small portion of the direction to the currently best candidate as a semi-deterministic update. DE starts with a population of $N_{p}$ number of vectors randomly chosen from the solution space. The vectors are updated in each iteration using two components, one being stochastic mutation component and the other being the semi-deterministic component. In each iteration, among all the vectors from the current and updated population, $N_{p}$ vectors with the least value of cost function are retained. Thus, it is expected that DE converges to a population whose best vector represents the global minimum. DE is controlled by the manipulation of three key parameters (i) population size, (ii) cross over and (iii) selection ratio. The basic difference between other population-based metaheuristic techniques (particularly genetic algorithm) and DE is the not so random mutation operation that is described below. In the present study, the population size is considered to be 100. For example, let us consider a member, $\bar{s}_{p, i}$ in the $i^{\text {th }}$ population. The mutation process for this affiliate can be 
expressed as follows [15],

$$
\bar{s}_{p, i}=\bar{d} \otimes \bar{s}_{p, i} \oplus \sim \bar{d} \otimes\left\{\bar{s}_{p, i}+c_{m}\left(\bar{s}_{\text {best }, i}-\bar{s}_{r, i}\right)\right\} ; \quad r \neq p, \text { best } .
$$

In the above, $\oplus, \otimes, \sim$ operators represent logical OR, AND, and NOT operatives, respectively. Additionally, $\bar{d}$ is randomly-chosen Boolean vector of the same size as $\bar{s}_{p, i}$ with probability described by a cross-over ratio $c_{r}$, which for the present work is considered to be 0.5 , whereas, $\bar{s}_{r, i}$ is a randomly-chosen member of the population. In $(2.20), c_{m}$ is referred to as the mutation probability (differential weight) which in the present work is taken as 1.0 and $\bar{s}_{\text {best }, i}$ is the member of the $i^{\text {th }}$ population for which the value of the relevant objective function is the least.

It is notable that the update scheme in (2.20) has the form of the original DE as follows $[1,15,27]$,

$$
\bar{s}_{p, i}=\bar{d} \otimes \bar{s}_{p, i} \oplus \sim \bar{d} \otimes\left\{\bar{a}+c_{m}(\bar{b}-\bar{c})\right\},
$$

where, $\bar{a}, \bar{b}, \bar{c}$ are randomly-chosen members of the current population. However, in $(2.21), \bar{a}$ and $\bar{b}$, are not randomly chosen but are represented by $\bar{a}=\bar{s}_{p, i}$, and $\bar{b}=\bar{s}_{\text {best }, i}$ respectively. In $(2.20)$, the mutation is directed to some degree by the preeminent member in the population and is not fully stochastic. In general, the entire population in the next iteration is stimulated by the best member of the current population in some or other manner, therefore, inculcating some deterministic or elitist characteristics. Inverse estimation is of great importance for an efficient design in any heat transfer problem. In the present study, the objective of inverse analysis is to predict the various unknown thermal parameters from a known stress field. As discussed before, the known stress field is obtained analytically using HPM method coupled with thermo-elasticity equation. To obtain the $n$ unknown parameters $\left(n \in \beta, N, \chi, G, E_{G}\right)$ inversely, the difference between the direct stress field and measured stress field is to be minimized iteratively. For this purpose, the following objective functions are used in the DE optimization for noise-free and noisy scenarios respectively,

$$
F=\sum_{j=1}^{p}\left\{\left(\sigma_{\phi_{j}}(n)-\bar{\sigma}_{\phi_{j}}\right) / \sigma_{\phi_{j}}\right\}^{2}, \quad F=\sum_{j=1}^{p}\left\{\left(\sigma_{\phi_{j}}(n)-\left[\bar{\sigma}_{\phi_{j}}+e_{r}\right]\right) / \sigma_{\phi_{j}}\right\}^{2}
$$

where $\bar{\sigma}_{\phi_{j}}$ is the direct tangential stresses obtained from the closed form forward solution of HPM and $\bar{\sigma}_{\phi_{j}}+e_{r}$ represents the same stress field with measurement error. The term, $\sigma_{\phi_{j}}$, is the guessed stress field estimated for a candidate vector in the solution space. This guessed stress field is computed for each vector in the current and updated populations of each iteration until the objective function is either below a threshold value, or saturates and does not improve over a few iterations, or the number of iterations exceed a maximum number of iterations. The subscript, $j$ denotes the number of measurement points along the fin's radius. Further details of optimization using DE can be found in the reference $[1,15]$. 


\section{Results and discussion}

The main purpose of this study is the inverse estimation of various unknown thermal parameters for an annular fin subjected to thermal stresses. It is well known that for an efficient and cost effective fin design, various thermal and geometric parameters are required to be optimised. Here, we present some numerical examples to illustrate the design using the proposed methodology.

Let us consider a situation where a fin should be designed in such a way that the maximum stresses due to the variation of heat transfer should not exceed certain limits. This condition helps to avoid the early damage of fin material from the thermal loading. With this intention, the various thermal parameters are estimated inversely using DE method from a prejudged stress field. The prejudged stress field is obtained from the HPM based forward solution viz. (2.17) and (2.18). The effects of thermal parameters on the non-dimensional temperature field are examined in Figure 2.

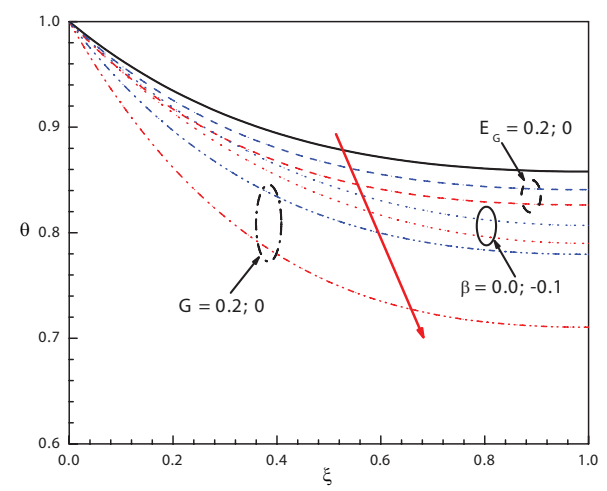

Figure 2. Effect of various non-dimensional thermal parameters, $G, E_{G}$, and $\beta$ on temperature distribution. Unless mention otherwise the values of parameters are, $G=0.4$, $E_{G}=0.4, \beta=0.3, \chi=1, N=1$.

It can be seen that the fin temperature gradually falls from its base to the tip, i.e. $\xi=0$ to $\xi=1$, and the temperature decays sharply near the base. The temperature field decreases with the decrease of thermal parameters, $\beta$, $G$, and $E_{G}$. It is worth mentioning that the parameter $\chi$ does not affect the temperature field, as it is connected with the stress distribution.

Figure 3 shows the contour plots representing the variation of temperature distribution as a function of the thermal parameters: (a) $\beta$, (b) $G$ and (c) $E_{G}$. It can be seen that in all cases the tip temperatures are monotonically increased with the increase of the parameters. Higher value of thermal conductivity parameter pertains to fast conduction process, as a result of which, high tip temperature is observed. Furthermore, higher tip temperatures can be observed with the increase of heat generation parameter.

The thermal stresses developed due to heat transfer through the fin are presented in Figure 4. It can be seen in Figure 4(a) that the magnitudes of radial stress (compressive) increases with the decrease of variable thermal conductivity parameter $\beta$. This is because of the thermal resistance increases 


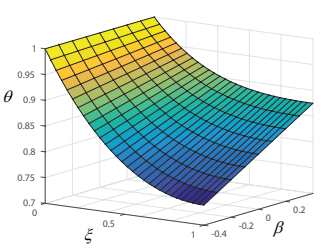

a)

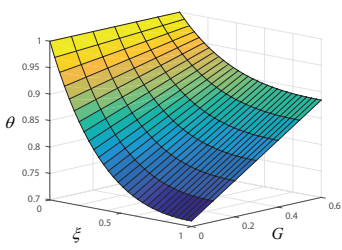

b)

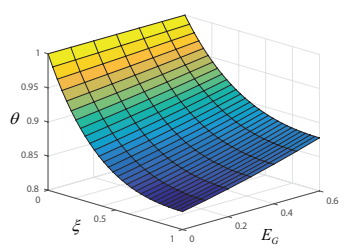

c)

Figure 3. Contour plots of temperature distribution as a function of thermal parameters. The values of parameters: (a) $N=1, G=0.4, E_{G}=0.4$ and $\beta=-0.4$ to 0.4 , (b) $N=1$, $\beta=0.3, E_{G}=0.0$ and $G=0$ to 0.6 , and (c) $N=1, \beta=0.3, G=0.4$ and $E_{G}=0$ to 0.6.

when the parameter $\beta$ decreases. As a result, the local free expansion of the fin material is obstructed. Interestingly, it can be seen that the radial stress fields are not influenced by the heat generation parameters $G$ and $E_{G}$. However, as shown in Figure 4(b), the tangential stress fields are influenced by all the thermal parameters. The influence of heat generation on the tangential stresses is minimum near to the base and gradually increases towards the fin tip.

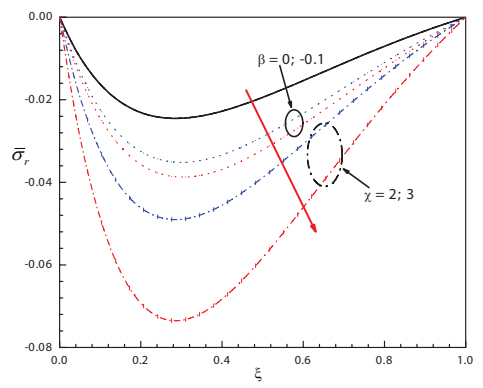

a)

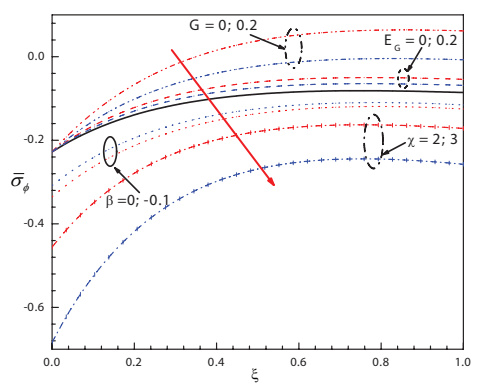

b)

Figure 4. Effect of various non-dimensional thermal parameters, $G, E_{G}$, $\beta$, and $\chi$ on (a) radial stress distribution and (b) tangential stress distribution. Unless mention otherwise the values of parameters are, $G=0.4, E_{G}=0.4, \beta=0.3, \chi=1, N=1$.

To show the effect of heat generation only, the variation of temperature and stress fields are plotted separately in Figure 5. The magnitude of radial and tangential stresses are directly related to the magnitude of coefficient of thermal expansion, $\chi$. The next step of our discussion is the inverse estimation of the various unknown thermal parameters. It can be seen that the tangential stress is significantly pronounced than the radial stress $\left(\bar{\sigma}_{\phi \mid \max } \approx 10 \bar{\sigma}_{r \mid \max }\right)$. As a result, it is expected that the thermal loading in the fin may cause material failure due to tangential stress only. Based on this, in the present analysis, the inverse parameters are estimated from the predefined tangential stress field, considering it as the reference stress field. For all the inverse simulations, the predefined tangential stress field is obtained from the forward solution of HPM, considering the values of parameters, $\beta=0.3, N=1.0, \chi=1.0, G=0.4$, and $E_{G}=0.4$. In the inverse simulations, $\beta, N$, and $\chi$ are considered the unknown variables. 


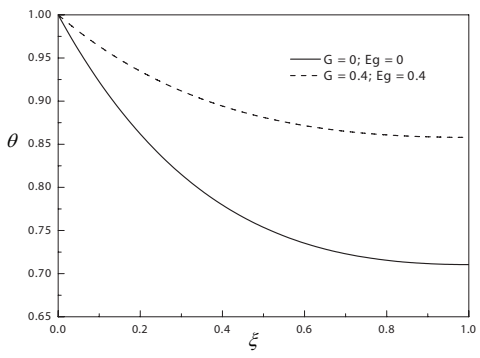

a)

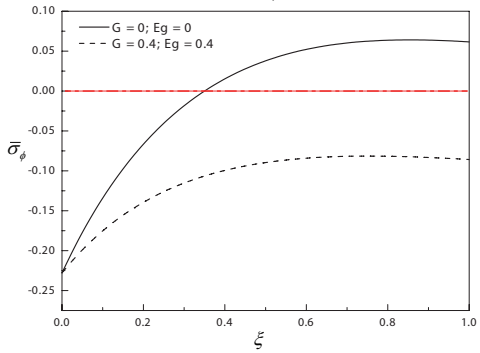

c)

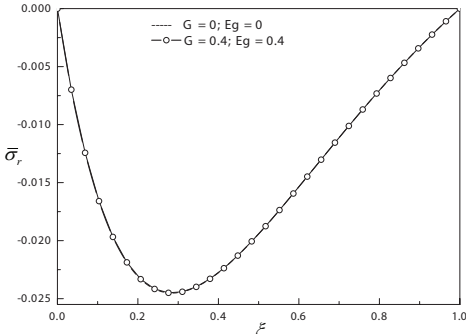

b)

Figure 5. Effect of heat generation parameter on the (a) temperature field, (b) radial stress field and (c) tangential stress field.

Table 1. Various combinations of three parameters inversely estimated using DE considering zero measurement error. Range: $[\beta, N, \chi]=[0-0.5 ; 0.6-1.4 ; 0.5-3]$.

\begin{tabular}{lllllll}
\hline Process & $\beta$ & $N$ & $\chi$ & $F(\xi)$ & Temp.(tip) & Efficiency \\
\hline Direct & 0.3 & 1 & 1 & $<10^{-3}$ & 0.7105 & 0.7530 \\
Run-1 & 0.2247 & 1.069 & 0.8775 & $<10^{-3}$ & 0.6699 & 0.7083 \\
Run-2 & 0.4056 & 0.8949 & 1.2448 & $<10^{-3}$ & 0.7676 & 0.8156 \\
Run-3 & 0.4785 & 0.8142 & 1.5 & $<10^{-3}$ & 0.8073 & 0.8592 \\
\hline
\end{tabular}

Table 1 presents the estimated values of three inverse parameters, $\beta, N$, and $\chi$ estimated using DE. The lower bound and upper bound of the parameters used in the simulation are mentioned in the table. The corresponding fin efficiencies obtained from the direct solution and inverse solutions are also presented in the table. For the correctness of the inverse parameters (Run 1 to Run 3), the tangential and radial stress fields are reconstructed using those parameters and compared with the direct stress field in Figure 6 .

It can be seen that the data points of direct vs. inverse stress fields make a $45^{\circ}$ line. This result suggests that the stress field obtained from the inverse parameters agrees with the reference stress field.

Now, we consider a more challenging inverse problem in which five thermal parameters, $\beta, N, \chi, G$ and $E_{G}$ are considered to be unknown. These five parameters are simultaneously estimated using DE, and the different combinations of their values are obtained in different runs. Table 2 presents the estimated values of five thermal parameters, $\beta, N, \xi, G$ and $E_{G}$, obtained 


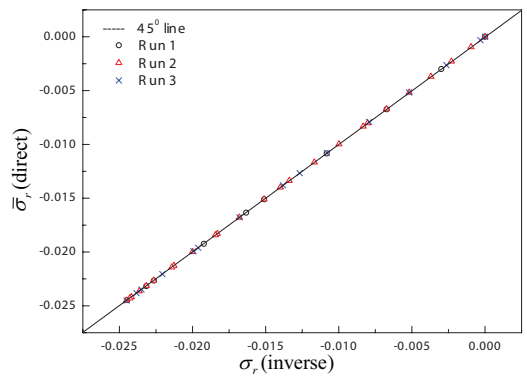

a)

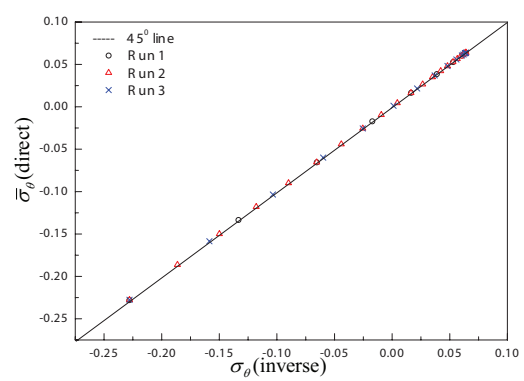

b)

Figure 6. Direct stress field vs. inverse stress field considering three inverse parameters $(N, \beta, \chi)$ estimation for (a) Radial stress and (b) Tangential stress.

Table 2. Various combinations of five parameters inversely estimated using DE considering zero measurement error. Range: $\left[\beta, N, G, E_{G}, \chi\right]=[0-0.5 ; 0.6-1.4 ; 0.05-0.8,0.05-0.8$, $0.5-3.0]$.

\begin{tabular}{llllllllll}
\hline Process & $\beta$ & $N$ & $G$ & $E_{G}$ & $\chi$ & $F(\xi)$ & $\begin{array}{l}\sigma_{\theta} \\
(\max .)\end{array}$ & $\begin{array}{l}\text { Temp. } \\
(\text { Tip })\end{array}$ & Efficiency \\
\hline Direct & 0.3 & 1.0 & 0.4 & 0.4 & 1.0 & $<10^{-3}$ & -0.0815 & 0.857 & 0.753 \\
Run-1 & 0.378 & 0.919 & 0.327 & 0.435 & 1.174 & $<10^{-3}$ & -0.0815 & 0.879 & 0.801 \\
Run-2 & 0.43 & 0.83 & 0.257 & 0.475 & 1.386 & $<10^{-3}$ & -0.0814 & 0.897 & 0.851 \\
Run-3 & 0.475 & 0.803 & 0.227 & 0.565 & 1.523 & $<10^{-3}$ & -0.0815 & 0.902 & 0.865 \\
\hline
\end{tabular}

using DE. The corresponding fin efficiencies are estimated using (2.19). These five parameters are simultaneously estimated using DE, and the different combinations of their values are obtained in different runs. Using these estimated parameters, the new temperature field and radial and tangential stress fields may be reconstructed using (2.10), (2.17), and (2.18) respectively. In Table 2, it can be seen that the maximum stresses obtained from the inverse parameters of all the runs are same as the reference ones. However, the fin tip temperatures obtained from the inverse parameters are different from the direct one and truly depend on the value of inversely-estimated parameters. All three runs generally indicate that various combinations of five parameters inverselyestimated using DE can meet a given maximum stress criterion. However, each combination yields a different temperature distribution that in turn results in different values of the fin efficiency. These results reveal that the efficiency of fin can be improved by optimizing the fin parameters without causing excess stress in the fin material.

Figure 7 depicts the reconstructed temperature and stress (radial and tangential) fields. As the inverse simulations were performed based on the forward solution of tangential stress field, the reconstructed temperature fields do not match with the direct temperature field. However, both the stress fields (radial and tangential) agree with the reference stress fields, as expected.

The convergence histories of the objective function and the iterative variation of all the five parameters with zero measurement error in the forward 


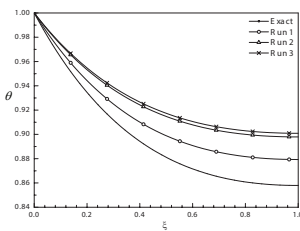

a)

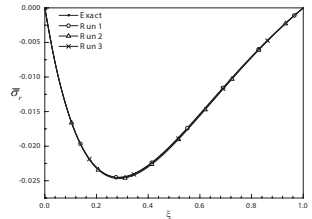

b)

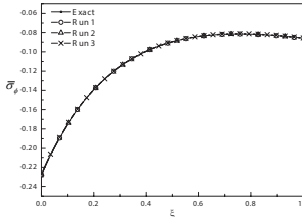

c)

Figure 7. Comparison between the direct (exact) and reconstructed (inverse) (a) temperature fields, (b) radial stress fields, and (c) tangential stress fields with no measurement error.

solution are presented in Figure 8. It can be seen that in all the cases the values of objective functions gradually reduces with the increasing number of iterations or generations (Figure 8(a)). However, the values of the five unknown parameters undergo random adjustment with the iterations. In most cases, the values of the unknown parameters stabilize approximately after 60 iterations there is insignificant update between 70-100 iterations. So, for the present work, 100 iterations of DE are satisfactory.

Table 3. Various combinations of five parameters inversely estimated using DE considering $\pm 15 \%$ random error in the forward solution. Range: $\left[\beta, N, G, E_{G}, \chi\right]=[-0.3-0.5 ; 0.6-1.4$; $0.05-0.8,0.05-0.8,0.5-3.0]$.

\begin{tabular}{llllllllll}
\hline Process & $\beta$ & $N$ & $G$ & $E_{G}$ & $\chi$ & $F(\xi)$ & $\begin{array}{l}\sigma_{\theta} \\
(\max .)\end{array}$ & $\begin{array}{l}\text { Temp. } \\
(\text { Tip })\end{array}$ & Efficiency \\
\hline Direct & 0.3 & 1.0 & 0.4 & 0.4 & 1.0 & $<0.02$ & -0.0815 & 0.857 & 0.7530 \\
Run-1 & 0.236 & 0.809 & 0.289 & 0.243 & 1.115 & $<0.02$ & -0.0794 & 0.8818 & 0.8433 \\
Run-2 & 0.334 & 0.861 & 0.256 & 0.644 & 1.142 & $<0.02$ & -0.0798 & 0.8835 & 0.8510 \\
Run-3 & -0.146 & 1.161 & 0.697 & 0.258 & 0.535 & $<0.02$ & -0.0790 & 0.7547 & 0.5845 \\
\hline
\end{tabular}

In real situation, the actual stress field developed in the fin slightly differs from the theoretical values due to measurement error. Thus, it is important to consider the estimation of unknown parameters in the presence of measurement errors in the stress field. Table 3 presents the estimated values of the unknown parameters for three different runs, obtained inversely from a prespecified stress field with $\pm 15 \%$ random measurement error in this case too, different combinations are observed in different runs. All three runs in general and run 3 in particular revel that diverse combinations of five inverselyestimated parameters yield a given stress distribution, but result in different temperature distributions. Consequently, they result in different values of the fin efficiency. Corresponding temperature of the fin's tip and the thermal efficiency are also presented in the table. It is observed that the DE saturates at higher values of the objective function in the presence of measurement error.

Using the inverse parameters of Table 3, the stress fields are reconstructed and compared with the reference stress field in Figure 9. In this case, the reconstructed stress fields differ slightly from the reference stress field. 


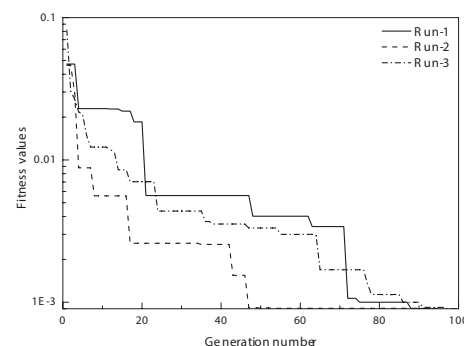

a)

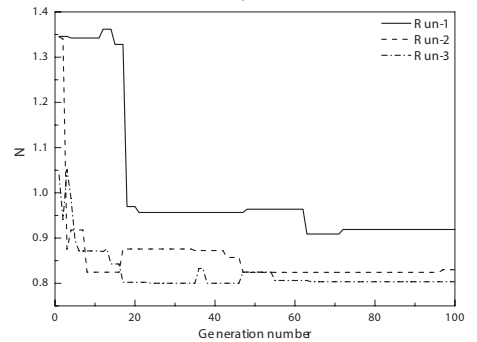

c)

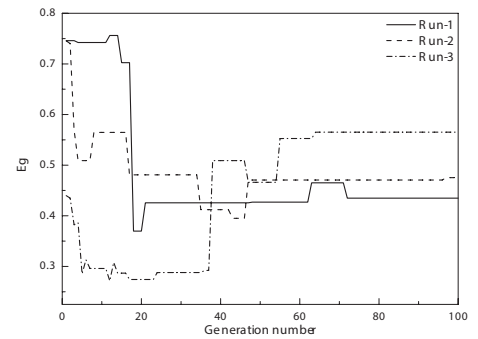

e)

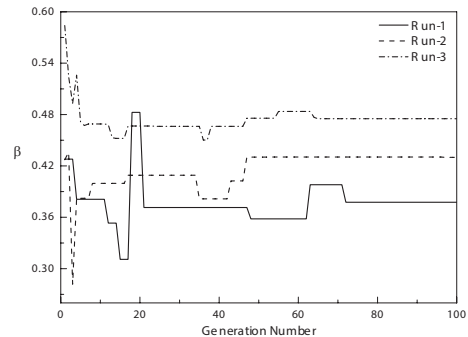

b)

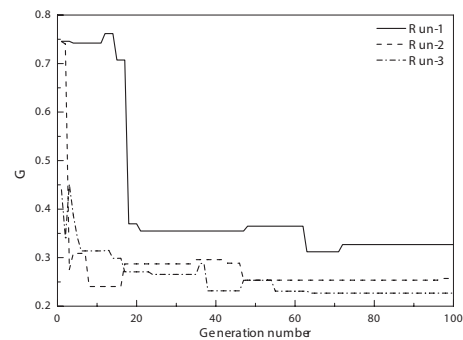

d)

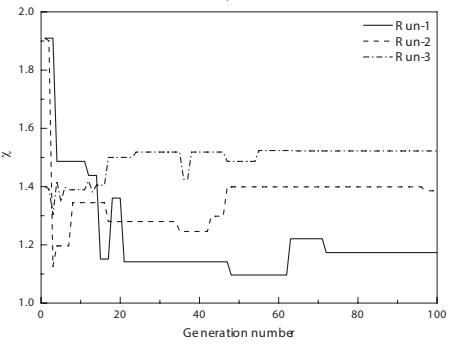

f)

Figure 8. Simultaneous estimation of various non-dimensional inverse thermal parameters of an annular fin subjected to thermal loading: (a) Fitness value of DE simulation without measurement error, (b) thermal conductivity parameter $(\beta),(\mathrm{c})$ thermo geometric parameter $(N)$, (d) heat generation number $(G),(\mathrm{e})$ heat generation parameter $\left(E_{G}\right)$, and (f) coefficient of thermal expansion $(\chi)$.

\section{Conclusions}

In this study, various unknown thermal parameters of an annular fin with variable thermal conductivity and internal heat generation have been inversely estimated, assuming that the thermal load in the fin will not cause excess stress field. The HPM has been successfully applied to obtain the temperature field in an annular fin with variable thermal conductivity and internal heat generation. The forward solution for the stress field is obtained directly from the classical theory of elasticity coupled with HPM based analytical solution of heat transfer. The study suggests that the variable thermal conductivity parameter influences the heat transfer as well as the thermal stresses. However, the internal heat generation phenomenon does not affect the radial stress. The effect of heat 


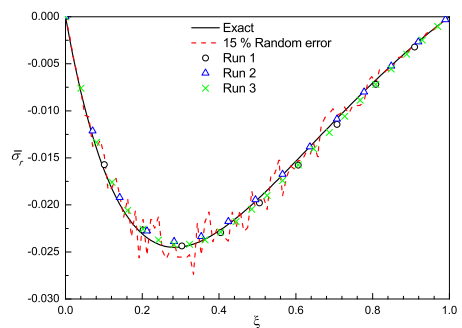

a)

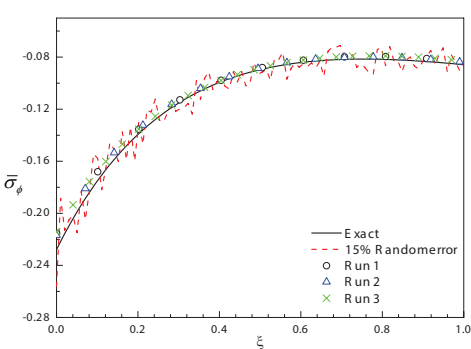

b)

Figure 9. Comparison between the direct (exact) and reconstructed (inverse) (a) radial stress fields, and (b) tangential stress fields. The inverse data obtained considering $15 \%$ random measurement error in direct data.

generation on the tangential stresses is minimum near to the base of the fin and increases nonlinearly towards the fin's tip. Differential evolution has been employed for the inverse solution of stress field. The predicted/reconstructed stress fields obtained from estimating all the thermal parameters using DE agree well with the exact stress fields. Very small difference in the reconstructed stress field is found when inverse solutions are obtained considering measurement error in the forward stress field.

\section{References}

[1] K. Agarwal and X. Chen. Application of differential evolution in 2-dimensional electromagnetic inverse problems. In Evolutionary Computation, 200\%. CEC 200\%. IEEE Congress on, pp. 4305-4312, 2007.

[2] İ.G. Aksoy. Adomian decomposition method for heat conduction in an annular fin of hyperbolic profile with temperature dependent thermal conductivity. ISI Bilimi Ve Teknigi Dergisi-Journal Of Thermal Science And Technology, 33(2):18, 2013.

[3] Z. Ayati and J. Biazar. On the convergence of homotopy perturbation method. Journal of the Egyptian Mathematical Society, 23(2):424-428, 2015. http://dx.doi.org/10.1016/j.joems.2014.06.015.

[4] A. Aziz and M.N. Bouaziz. A least squares method for a longitudinal fin with temperature dependent internal heat generation and thermal conductivity. Energy Conversion and Management, 52(8):2876-2882, 2011.

[5] J. Biazar and H. Aminikhah. Study of convergence of homotopy perturbation method for systems of partial differential equations. Computers \& Mathematics with Applications, 58(11-12):2221-2230, 2009. http://dx.doi.org/10.1016/j.camwa.2009.03.030.

[6] A.P. Boresi, K. Chong and J.D. Lee. Elasticity in engineering mechanics. John Wiley \& Sons, 2010. http://dx.doi.org/10.1002/9780470950005.

[7] F. Chabane, N. Moummi and S Benramache. Experimental study of heat transfer and thermal performance with longitudinal fins of so- 
lar air heater. Journal of Advanced Research, 5(2):183-192, 2014. http://dx.doi.org/10.1016/j.jare.2013.03.001.

[8] W.-L. Chen, Y.-C. Yang and H.-L. Lee. Inverse problem in determining convection heat transfer coefficient of an annular fin. Energy Conversion and Management, 48(4):1081-1088, 2007. http://dx.doi.org/10.1016/j.enconman.2006.10.016.

[9] C.-H. Chiu and C.-K. Chen. Application of the decomposition method to thermal stresses in isotropic circular fins with temperaturedependent thermal conductivity. Acta Mechanica, 157(1):147-158, 2002. http://dx.doi.org/10.1007/BF01182160.

[10] C.-H. Chiu and C.-K. Chen. Application of Adomians decomposition procedure to the analysis of convective-radiative fins. Journal of Heat Transfer, 125(2):312316, 2003. http://dx.doi.org/10.1115/1.1532012.

[11] M.S.H. Chowdhury, I. Hashim and O. Abdulaziz. Comparison of homotopy analysis method and homotopy-perturbation method for purely nonlinear fin-type problems. Communications in Nonlinear Science and Numerical Simulation, 14(2):371-378, 2009. http://dx.doi.org/10.1016/j.cnsns.2007.09.005.

[12] S.B. Coşkun and M.T. Atay. Fin efficiency analysis of convective straight fins with temperature dependent thermal conductivity using variational iteration method. Applied Thermal Engineering, 28(17-18):2345-2352, 2008. http://dx.doi.org/10.1016/j.applthermaleng.2008.01.012.

[13] M. Cumo, S. Lopez and G.C. Pinchera. Numerical calculation of extended surface efficiency. In Chemical engineering progress symposium series, volume 61, pp. 225-233, 1965.

[14] R Das. Forward and inverse solutions of a conductive, convective and radiative cylindrical porous fin. Energy Conversion and Management, 87:96-106, 2014. http://dx.doi.org/10.1016/j.enconman.2014.06.096.

[15] R. Das and D.K. Prasad. Prediction of porosity and thermal diffusivity in a porous fin using differential evolution algorithm. Swarm and Evolutionary Computation, 23:27-39, 2015. http://dx.doi.org/10.1016/j.swevo.2015.03.001.

[16] M. Hatami, A. Hasanpour and D.D. Ganji. Heat transfer study through porous fins ( $\mathrm{Si} 3 \mathrm{~N} 4$ and $\mathrm{Al}$ ) with temperature-dependent heat generation. Energy Conversion and Management, 74:9-16, 2013. http://dx.doi.org/10.1016/j.enconman.2013.04.034.

[17] J He. An approximate solution technique depending on an artificial parameter: A special example. Communications in Nonlinear Science and Numerical Simulation, 3(2):92-97, 1998.

[18] J.-H. He. Homotopy perturbation method for solving boundary value problems. Physics letters A, 350(1-2):87-88, 2006. http://dx.doi.org/10.1016/j.physleta.2005.10.005.

[19] A.D. Kraus, A. Aziz and J Welty. Extended Surface Heat Transfer. John Wiley \& Sons, 2002.

[20] B. Kundu and P.K. Das. Optimum profile of thin fins with volumetric heat generation: a unified approach. Journal of Heat Transfer, 127(8):945-948, 2005. http://dx.doi.org/10.1115/1.1929784.

[21] A. Mallick and R. Das. Application of simplex search method for predicting unknown parameters in an annular fin subjected to 
thermal stresses. Journal of Thermal Stresses, 37(2):236-251, 2014. http://dx.doi.org/10.1080/01495739.2013.839850.

[22] A. Mallick, S. Ghosal, P.K. Sarkar and R. Ranjan. Homotopy perturbation method for thermal stresses in an annular fin with variable thermal conductivity. Journal of Thermal Stresses, 38(1):110-132, 2015. http://dx.doi.org/10.1080/01495739.2014.981120.

[23] G.B. Melese and J.E. Wilkins. Efficiency of longitudinal fins of arbitrary shape with nonuniform surface heat transfer and internal heat generation. In Chemical Engineering Progress, volume 62, p. 81, 1966.

[24] S. Mosayebidorcheh, M. Farzinpoor and D.D. Ganji. Transient thermal analysis of longitudinal fins with internal heat generation considering temperaturedependent properties and different fin profiles. Energy Conversion and Management, 86:365-370, 2014. http://dx.doi.org/10.1016/j.enconman.2014.05.033.

[25] R.K. Singla and R Das. Application of Adomian decomposition method and inverse solution for a fin with variable thermal conductivity and heat generation. International Journal of Heat and Mass Transfer, 66:496-506, 2013. http://dx.doi.org/10.1016/j.ijheatmasstransfer.2013.07.053.

[26] D. Soodphakdee, M. Behnia and D.W. Copeland. A comparison of fin geometries for heatsinks in laminar forced convectionpart i: Round, Elliptical, and Plate fins in staggered and in-line configurations. The International Journal of Microcircuits and Electronic Packaging, 24(1):68-76, 2001.

[27] R. Storn and K. Price. Differential evolution-a simple and efficient heuristic for global optimization over continuous spaces. Journal of Global Optimization, 11(4):341-359, 1997. http://dx.doi.org/10.1023/A:1008202821328.

[28] S.P. Timoshenko and J.N. Goodier. Theory of elasticity. International Journal of Bulk Solids Storage in Silos, 1(4), 2014.

[29] S.-S. Wu. Analysis on transient thermal stresses in an annular fin. Journal of Thermal Stresses, 20(6):591-615, 1997. http://dx.doi.org/10.1080/01495739708956120.

[30] Y.-C. Yang and S.-S. Chu. Transient coupled thermoelastic analysis of an annular fin. International Communications in Heat and Mass Transfer, 28(8):1103-1114, 2001. http://dx.doi.org/10.1016/S0735-1933(01)00313-X. 